\title{
Correction to: Coupled nature of evening-time ionospheric electrodynamics
}

\author{
L.M. Joshi ${ }^{1,2} \cdot$ L.C. Tsai ${ }^{2}$
}

Published online: 5 April 2018

๑) Springer Science+Business Media B.V., part of Springer Nature 2018

\section{Correction to: Astrophys Space Sci (2018) 363:72 https://doi.org/10.1007/s10509-018-3288-z}

It is to be brought to the notice of the readers that an error was made while plotting Figs. 3 and 4. This happened due to wrong folders being chosen for plotting the Es parameters corresponding to the low and high PRE cases. High Es and low Es occurrence cases were plotted instead of the Es conditions corresponding to the low and high PRE cases. This error happened unintentionally and is being brought to the notice of the readers promptly. In order to resolve the issue, the entire scaling of the ionograms has been redone and Figs. 3 and 4 have been plotted again with the new scaled data. The new Fig. 3 shows the ftEs, fbEs and occurrence of the Es in high (Q4) and low (Q1) PRE cases. The new Fig. 4 shows the average $\mathrm{E}$ region electron density in the Q1 and Q4 cases. The method of calculating the average E region electron density in the new Fig. 4 is the same as that in the old Fig. 4. The broad scientific implications are as follows: a difference in the Es conditions has been observed in the Q1 and Q4 cases in early post sunset hours in the new Fig. 3 too; however, this difference is not as remarkable as in the old Fig. 3. The occurrence frequency of Esb in Q1 cases at 18 IST is higher than that in Q4 cases in the new Fig. 3 also; however, this occurrence is not $100 \%$ as in the old Fig. 3. Thus the suppression of the PRE due to

The online version of the original article can be found under https://doi.org/10.1007/s10509-018-3288-z.

\footnotetext{
L.M. Joshi

lmjoshinarl@gmail.com

1 Indian Institute of Geomagnetism, Navi Mumbai, India

2 Center for Space and Remote Sensing Research, National Central University, Chung-Li, Taiwan, ROC
}

the occurrence of Es at low latitude is not fully conclusive. A more comprehensive investigation with a larger dataset will be carried out in future to investigate the PRE-Es relationship. Although the present result gives evidence for the role Es can play in suppressing the PRE, the evidence is not fully conclusive. 
Fig. 3 Es parameters

corresponding to high (Q4) and low (Q1) PRE cases.

$(\mathbf{a}-\mathbf{c})$ indicates top frequency of Es (ftEs), blanketing frequency of Es (fbEs), and the occurrence frequency of the Es/Esb, respectively, for high (Q4) PRE cases. $(\mathbf{d}-\mathbf{f})$ indicates the same but corresponding to low (Q1) PRE cases. Details are given in the text

Fig. 4 Background peak $\mathrm{E}$ region electron densities in low PRE, Q1 (solid line) and high PRE, Q4 (dotted line) cases
Es parameters corresponding to high PRE cases

(a)

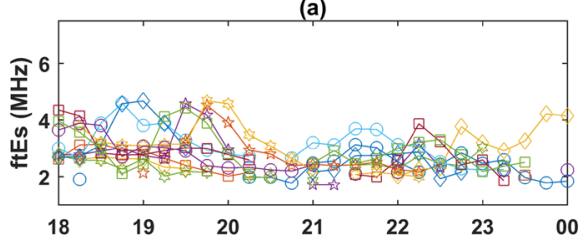

(b)

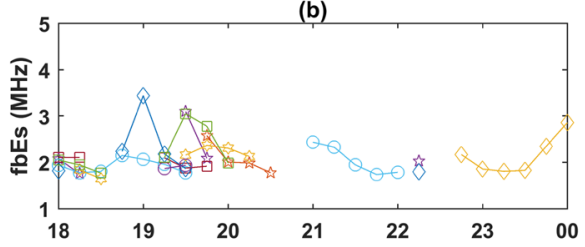

(c)

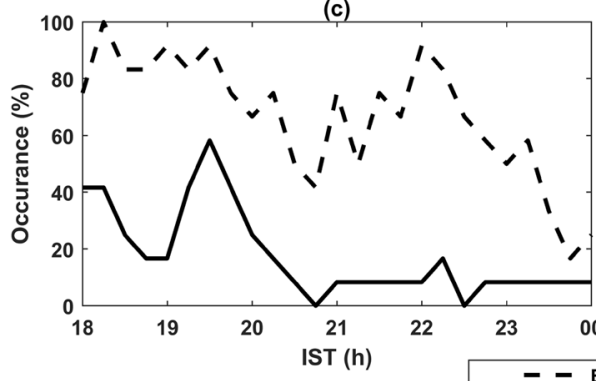

Es parameters corresponding to low PRE cases

(d)

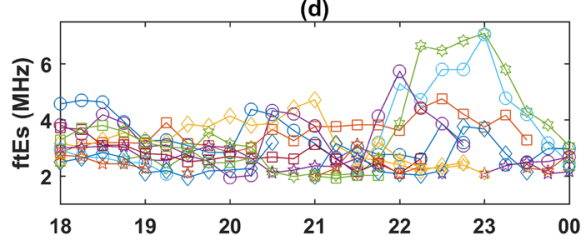

(e)

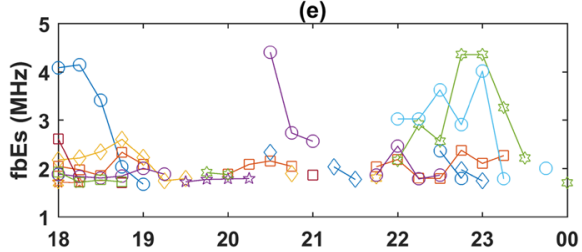

(f)

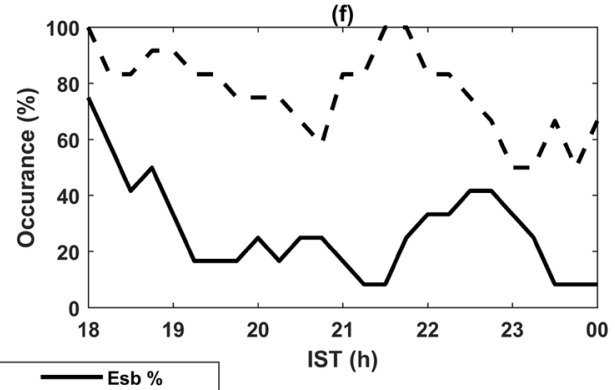

E region electron density in Q1 and Q4 cases

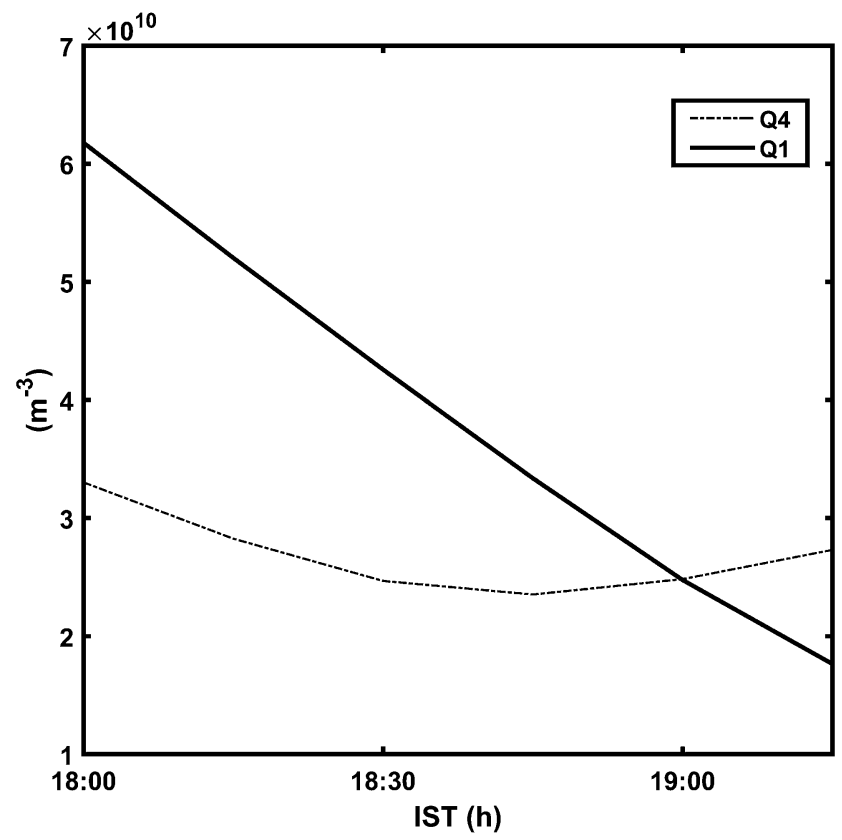

